\title{
Measurements of the properties of the Higgs boson using the ATLAS detector
}

\author{
Michaela Queitsch-Maitland ${ }^{1, a}$, On behalf of the ATLAS Collaboration. \\ ${ }^{1}$ Department of Physics \& Astronomy, University of Manchester, Oxford Road, Manchester, M13 9PL, UK.
}

\begin{abstract}
Measurements of the properties of the Higgs boson with the ATLAS detector are presented. The spin, parity and tensor couplings of the Higgs boson in the $H \rightarrow \gamma \gamma$, $H \rightarrow Z Z^{*} \rightarrow 4 \ell$ and $H \rightarrow W W^{*} \rightarrow e v \mu \nu$ channels are investigated. The results are based on $p p$ collision data collected by the ATLAS detector corresponding to an integrated luminosity of $4.7 \mathrm{fb}^{-1}$ at centre-of-mass energy $\sqrt{s}=7 \mathrm{TeV}$, and $20.3 \mathrm{fb}^{-1}$ at $\sqrt{s}=8 \mathrm{TeV}$. Measurements of the fiducial and differential cross sections for Higgs boson production in the $H \rightarrow \gamma \gamma$ and $H \rightarrow Z Z^{*} \rightarrow 4 \ell$ are also presented using $20.3 \mathrm{fb}^{-1}$ of $\sqrt{s}=8 \mathrm{TeV}$ data. The cross section measurements in the $H \rightarrow \gamma \gamma$ channel are then used to set limits on anomalous Higgs boson interactions in an effective Lagrangian. All results are consistent with expectations for a Standard Model Higgs boson.
\end{abstract}

\section{Introduction}

The discovery of a Higgs boson [1, 2] in 2012 by the ATLAS [3] and CMS [4] Collaborations at the Large Hadron Collider (LHC) marked an exciting new era in experimental particle physics. With more data, emphasis has now shifted to detailed analysis of the properties of the new particle. These measurements are crucial to determine whether this new particle is indeed the Higgs boson as predicted by the Standard Model (SM), or whether it is a more exotic Higgs boson described by a theory beyond the Standard Model (BSM).

This note presents a selection of measurements and their interpretations that probe different properties of Higgs boson production. The compatibility of the data with different spin, parity and tensor coupling scenarios of the Higgs boson are tested in the $H \rightarrow \gamma \gamma, H \rightarrow Z Z^{*} \rightarrow 4 \ell$ and $H \rightarrow W W^{*} \rightarrow e v \mu \nu$ decay channels. Measurements of fiducial and differential cross section for Higgs boson production in the $H \rightarrow \gamma \gamma, H \rightarrow Z Z^{*} \rightarrow 4 \ell$ decay channels are also presented. Lastly an interpretation of the measured differential cross sections in the $H \rightarrow \gamma \gamma$ channel, using an effective Lagrangian to probe anomalous Higgs boson couplings, is shown. The results are based on $p p$ collision data collected by the ATLAS detector, and correspond to an integrated luminosity of $4.7 \mathrm{fb}^{-1}$ at centre-of-mass energy $\sqrt{s}=7 \mathrm{TeV}$, and $20.3 \mathrm{fb}^{-1}$ at $\sqrt{s}=8 \mathrm{TeV}$.

\section{Spin and parity interpretations}

In the SM the Higgs boson is a CP-even scalar particle, $J^{C P}=0^{++}$. Theories beyond the SM may introduce Higgs bosons with different spin-CP structure, and may include CP-mixing. The Higgs char-

ae-mail: michaela.queitsch-maitland@cern.ch 
acterisation framework [5], which uses an effective field theory (EFT) approach, is used to confront the data with different spin-parity scenarios. The SM hypothesis $J^{P}=0^{+}$is compared to alternative models including a pseudo-scalar boson $\left(J^{P}=0^{-}\right)$, a BSM scalar boson $\left(J^{P}=0_{h}^{+}\right)$, and a graviton-like tensor model $\left(J^{P}=2^{+}\right)$with universal $\left(\kappa_{q}=\kappa_{g}\right)$ and non-universal $\left(\kappa_{q} \neq \kappa_{g}\right)$ couplings.

The measurements $[6,7]$ perform fits of discriminant observables sensitive to the spin and parity of the signal. For $H \rightarrow \gamma \gamma$ the data are divided into categories based on $\left|\cos \theta^{*}\right|$, the production angle of the two photons in the Collins-Soper reference frame [8], and the $p_{\mathrm{T}}$ of the diphoton system. The $H \rightarrow Z Z^{*} \rightarrow 4 \ell$ channel uses a matrix element based discriminant. For the $H \rightarrow W W^{*} \rightarrow e v \mu \nu$ decay channel a Boosted Decision Tree (BDT) for each model is trained with observables based on the lepton kinematics and missing transverse energy.

The test statistic, $\tilde{q}$, that is used to distinguish between two spin-parity hypotheses is based on a ratio of profile likelihoods evaluated under either the SM or alternative spin-parity hypothesis. All considered non-SM spin hypotheses, shown in Figure 1, are excluded at more than $99.9 \% \mathrm{CL}_{\mathrm{s}}$.

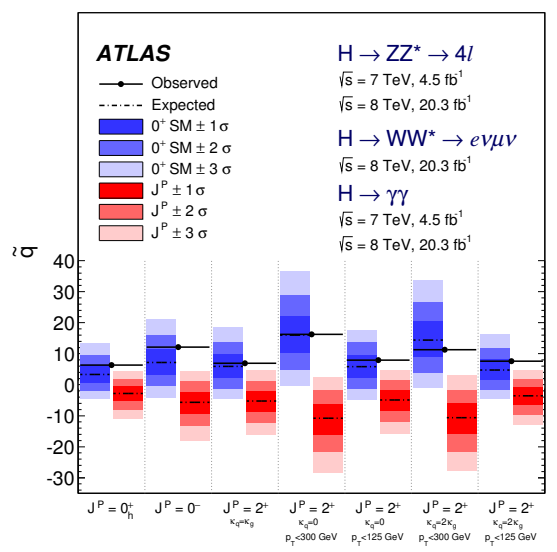

Figure 1. Distributions of the test statistic for the SM Higgs boson (blue) and alternative $\mathrm{J}^{\mathrm{P}}$ hypothesis (red). The observed values are indicated by the black points. These fits are performed combining the $H \rightarrow \gamma \gamma, H \rightarrow Z Z^{*} \rightarrow$ $4 \ell$ and $H \rightarrow W W^{*} \rightarrow e v \mu \nu$ decay channels. See Auxiliary Material of [7].

In addition to fixed spin and parity tests, which probe only one $\mathrm{CP}$ eigenstate, the tensor structure of the HVV interaction was also investigated. This is done assuming a spin- 0 hypothesis and testing a mixture of SM + BSM CP-even and SM + BSM CP-odd independently. Limits are set on the parameters $\kappa_{\mathrm{HVV}} / \kappa_{\mathrm{SM}}$ and $\left(\kappa_{\mathrm{AVV}} / \kappa_{\mathrm{SM}}\right) \tan \alpha$. The likelihood scans of these parameters are shown in Figure 2. The best fit values for the parameters are within the SM expectation.

\section{Fiducial and differential cross sections}

The measurement of fiducial and differential cross sections for Higgs boson production offer an alternative way of probing the properties of the Higgs boson. Cross sections allow a wide range of physics to be investigated, such as the theoretical modelling of gluon-fusion production, spin-CP, and associated jet activity. Fiducial cross sections are defined within a fiducial volume, which is specified by physics object definitions and kinematic selection requirements. The fiducial cross section for a fiducial region, $i$, is given by 

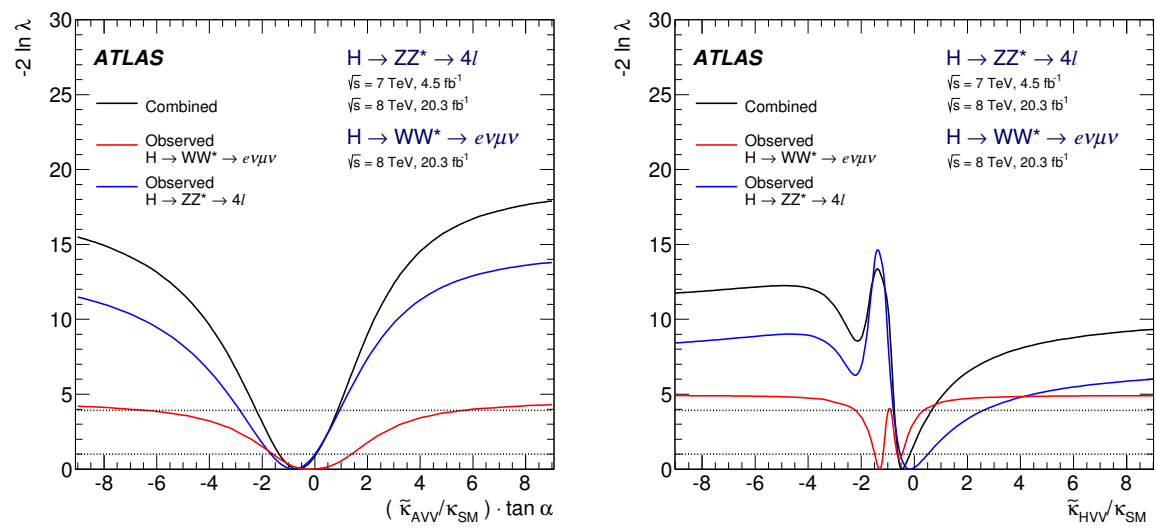

Figure 2. Observed distribution for the test statistic for the $H \rightarrow Z Z^{*} \rightarrow 4 \ell$ and $H \rightarrow W W^{*} \rightarrow$ ev $\mu \nu$ analyses and their combination, shown as a function of $\kappa_{\mathrm{HVV}} / \kappa_{\mathrm{SM}}$ and $\left(\kappa_{\mathrm{AVV}} / \kappa_{\mathrm{SM}}\right) \tan \alpha$. The $68 \%$ and $95 \% \mathrm{CL}$ exclusion regions are indicated by the corresponding horizontal lines [7].

$$
\sigma_{i}^{f i d}=\frac{v_{i}^{s i g}}{c_{i} \int L \mathrm{~d} t}
$$

where $c_{i}$ is a correction factor, which is determined using simulated Higgs boson event samples and accounts for the difference in yield between particle level and detector level. These correct for inefficiency and resolution effects of the detector. Therefore measurements of these fiducial cross sections can be interpreted in a wide variety of theoretical models at the particle level, and the theoretical uncertainties are mostly factorised from the measurement. The particle level selection criteria are chosen to be as close to the selection applied to the data as possible to minimise the extrapolation of phase space and to reduce uncertainties.

The measured fiducial cross sections for the $H \rightarrow \gamma \gamma$ [9] and $H \rightarrow Z Z^{*} \rightarrow 4 \ell$ [10] decay channels are

$$
\begin{aligned}
\sigma_{\gamma \gamma}^{f i d}=43.2 \pm 9.4(\text { stat. })_{-2.9}^{+3.2} \text { (syst.) } \pm 1.2 \text { (lumi.) } \mathrm{fb} \\
\sigma_{\text {LHC-XS }}^{f i d}=30.5 \pm 3.3 \mathrm{fb} \\
\sigma_{4 \ell}^{f i d}=2.11_{-0.47}^{+0.53} \text { (stat.) } \pm 0.08 \text { (syst.) fb } \\
\sigma_{\text {LHC-XS }}^{f i d}=1.3 \pm 0.13 \mathrm{fb}
\end{aligned}
$$

where $\sigma_{\mathrm{LHC}-\mathrm{XS}}^{\text {fid }}$ are the current best estimate of the predicted SM cross sections, in the respective fiducial regions and for a Higgs boson mass of $125.4 \mathrm{GeV}$. Details of the definitions of the fiducial regions can be found in $[9,10]$.

The differential cross sections are measured for many observables that are sensitive to various properties of the Higgs boson. For example, the $p_{\mathrm{T}}$ of the Higgs boson and the jet multiplicity, $N_{\text {jets }}$, 
shown in Figure 3 are sensitive to the theoretical modelling of gluon fusion as well as additional jet activity produced in association with the Higgs boson.
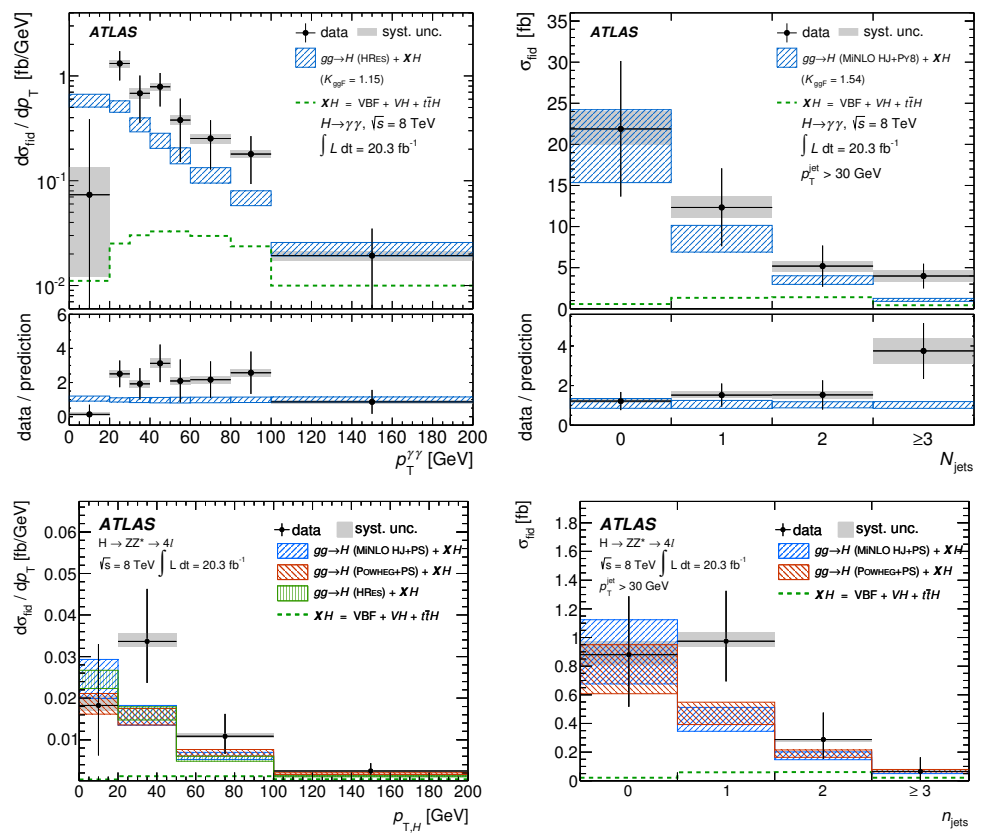

Figure 3. Measured differential cross sections as a function of the Higgs boson $p_{\mathrm{T}}$ (left) and $N_{\text {jets }}$ (right) for the $H \rightarrow \gamma \gamma$ (top) and $H \rightarrow Z Z^{*} \rightarrow 4 \ell$ (bottom) channels. The black points represent the data, where the total uncertainty is given by the vertical black bar and the systematic uncertainty by the grey band. The SM predictions are shown in coloured hatched boxes, with the width of the band representing the theoretical uncertainty $[9,10]$.

Inclusive cross sections,

$$
\sigma_{i}^{i n c l}=\frac{\sigma_{i}^{f i d}}{\alpha_{i} \mathcal{B}}=\frac{v_{i}^{s i g}}{\alpha_{i} \mathcal{B} c_{i} \int L \mathrm{~d} t}
$$

which are corrected for the acceptance of the fiducial phase space, $\alpha_{i}$, and the branching fraction, $\mathcal{B}$ are also extracted. In this way data in the $H \rightarrow \gamma \gamma$ and $H \rightarrow Z Z^{*} \rightarrow 4 \ell$ are combined to reduce statistical uncertainties through a likelihood fit. The resulting total cross section and prediction [11] are

$$
\begin{aligned}
\sigma_{\text {data }}^{p p \rightarrow H} & =33.0 \pm 5.3(\text { stat. }) \pm 1.6(\text { syst. }) \mathrm{pb} \\
\sigma_{\mathrm{LHC}-\mathrm{XS}}^{p p \rightarrow H} & =22.16 \pm 1.99(\text { scale }) \pm 2.00(\mathrm{pdf}) \mathrm{pb} .
\end{aligned}
$$

The normalised differential cross sections for the Higgs boson $p_{\mathrm{T}}$ and the leading jet $p_{\mathrm{T}}$ are shown in Figure 4. All cross section measurements are consistent with the expectations for a SM Higgs boson. 

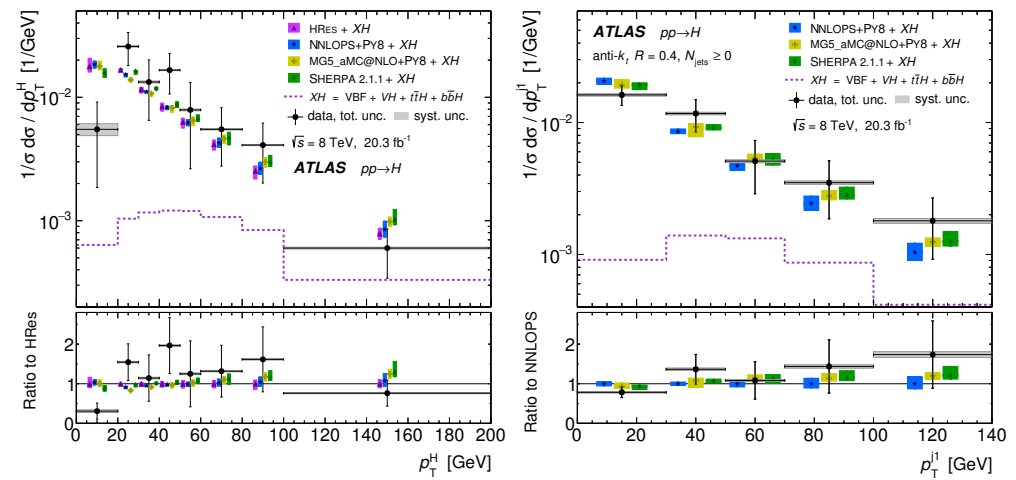

Figure 4. Normalised differential cross section for Higgs $p_{\mathrm{T}}$ (left) and leading jet $p_{\mathrm{T}}$ (right) combining data in the $H \rightarrow \gamma \gamma$ and $H \rightarrow Z Z^{*} \rightarrow 4 \ell$ channels. The data are compared to different state-of-the-art theoretical predictions [11].

\section{Interpretations of cross section measurements using an effective Lagrangian}

The cross section measurements in the $H \rightarrow \gamma \gamma$ channel discussed in Section 3 were also used to set limits on Wilson coefficients for the strongly interacting light Higgs (SILPH) EFT formalism [12]. The measurement uses five of the differential distributions in [9], where the statistical correlations between bins of observables in data are evaluated using pseudo-experiments [13]. An example for these correlations are shown in Figure 5 for diphoton $p_{\mathrm{T}}$ and $N_{\text {jets }}$.

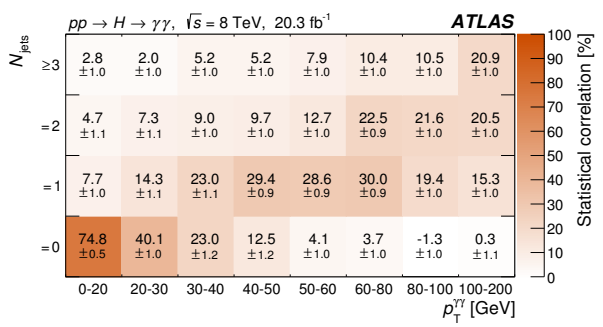

Figure 5. Statistical correlations between individual bins in diphoton $p_{\mathrm{T}}$ and $N_{\text {jets }}$ evaluated using a bootstrapping procedure. The errors represent the statistical precision of the pseudo-experiments and the data [13].

The Lagrangian is described by

$$
\begin{aligned}
\mathcal{L} & =\bar{c}_{\gamma} O_{\gamma}+\bar{c}_{g} O_{g}+\bar{c}_{\mathrm{HW}} O_{\mathrm{HW}}+\bar{c}_{\mathrm{HB}} O_{\mathrm{HB}} \\
& +\tilde{c}_{\gamma} O_{\gamma}+\tilde{c}_{g} O_{g}+\tilde{c}_{\mathrm{HW}} O_{\mathrm{HW}}+\tilde{c}_{\mathrm{HB}} O_{\mathrm{HB}}
\end{aligned}
$$

where $\bar{c}_{i}$ and $\tilde{c}_{i}$ are Wilson coefficients that specify the strength of new CP-even and CP-odd interactions respectively. The $O_{i}$ are dimension six operators described in [12]. The terms with $\gamma, g$, and HW or HB affect the coupling of the Higgs to photons, gluons, and weak vector bosons respectively. 
Limits are set on the Wilson coefficients by constructing a $\chi^{2}$ function with respect to data and each prediction. Scans of one or two Wilson coefficients are carried out at a time. The $68 \%$ and $95 \%$ confidence level (CL) regions for example scans are shown in Figure 6. No significant deviations from the SM predictions are observed, and limits are set on the Wilson coefficients.
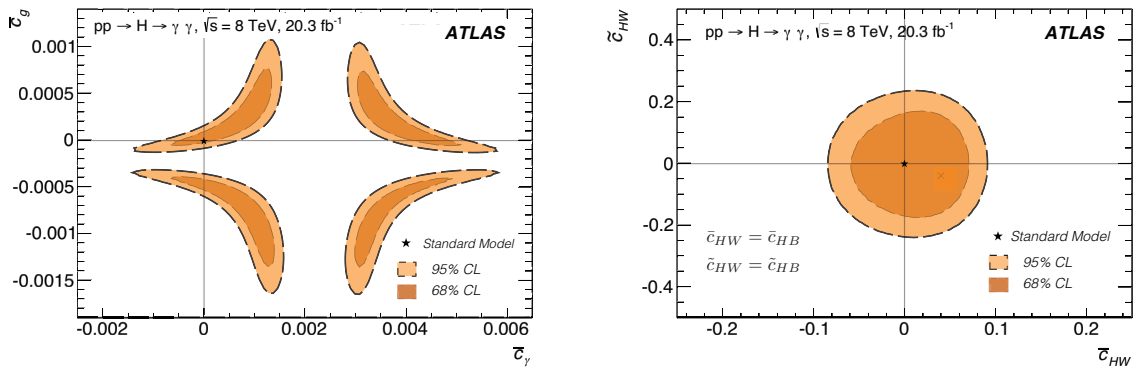

Figure 6. The $68 \%$ (dark) and $95 \%$ (light) confidence regions for fits to the $\bar{c}_{\gamma}$ and $\tilde{c}_{g}$ (left), and $\bar{c}_{\mathrm{HW}}$ and $\tilde{c}_{\mathrm{HW}}$ (right) Wilson coefficients. All other coefficients are set to zero, except for the fits to $\bar{c}_{\mathrm{HW}}$ and $\tilde{c}_{\mathrm{HW}}$ (right), where $\bar{c}_{\mathrm{HW}}=\bar{c}_{\mathrm{HB}}$ and $\tilde{c}_{\mathrm{HW}}=\tilde{c}_{\mathrm{HB}}$. The marker indicates the SM value [13].

\section{Summary and Conclusion}

Measurements probing the properties of the Higgs boson at the LHC are reported, based on $p p$ collision data collected by the ATLAS detector corresponding to an integrated luminosity of $4.7 \mathrm{fb}^{-1}$ at centre-of-mass energy $\sqrt{s}=7 \mathrm{TeV}$, and $20.3 \mathrm{fb}^{-1}$ at $\sqrt{s}=8 \mathrm{TeV}$. The compatibility of the data with different spin, parity and tensor coupling scenarios of the Higgs boson are tested in the $H \rightarrow \gamma \gamma$, $H \rightarrow Z Z^{*} \rightarrow 4 \ell$ and $H \rightarrow W W^{*} \rightarrow e v \mu \nu$ decay channels. Fiducial and differential cross sections for Higgs boson production in the $H \rightarrow \gamma \gamma, H \rightarrow Z Z^{*} \rightarrow 4 \ell$ decay channels are presented. In the $H \rightarrow \gamma \gamma$ channel a selection of the measured differential cross sections are used to set limits on anomalous Higgs boson couplings using an effective Lagrangian. No significant deviations from the SM predictions are observed in any of the measurements discussed.

\section{References}

[1] ATLAS Collaboration, Phys. Lett. B716, 1-29 (2012).

[2] CMS Collaboration, Phys. Lett. B716, 30-61 (2012).

[3] ATLAS Collaboration, JINST 3 S08003 (2008).

[4] CMS Collaboration, JINST 3 S08004 (2008).

[5] P. Artoisenet, et al., JHEP 11, 043 (2013).

[6] ATLAS Collaboration, Eur. Phys. J. C75, 231 (2015).

[7] ATLAS Collaboration, Eur. Phys. J. C75, 476 (2015).

[8] J. Collins and D. Soper, Phys. Rev. D 16, 2219 (1977).

[9] ATLAS Collaboration, JHEP 09, 112 (2014).

[10] ATLAS Collaboration, Phys. Lett. B738, 234-253 (2014). 
[11] ATLAS Collaboration, Phys. Rev. Lett. 115, 091801 (2015).

[12] R. Contino, et al., JHEP 1307, 035 (2013).

[13] ATLAS Collaboration, Phys. Lett. B753, 69-85 (2016). 